\title{
Hematopoietic Effects of Paeoniflorin and Albiflorin on Radiotherapy-Induced Myelosuppression Mice
}

\author{
Yingli Zhu, ${ }^{1}$ Linyuan Wang, ${ }^{1}$ Zhihui Yang, ${ }^{2}$ Jingxia Wang, ${ }^{1}$ Wei Li, \\ Jianyu Zhou, ${ }^{1}$ and Jianjun Zhang ${ }^{1}$ \\ ${ }^{1}$ Beijing University of Chinese Medicine, Beijing 100029, China \\ ${ }^{2}$ Department of Psychiatry \& Neuroscience, University of Florida, Gainesville, FL 32611, USA
}

Correspondence should be addressed to Jianjun Zhang; zhangjianjun@bucm.edu.cn

Received 5 February 2016; Accepted 8 May 2016

Academic Editor: I-Min Liu

Copyright (C) 2016 Yingli Zhu et al. This is an open access article distributed under the Creative Commons Attribution License, which permits unrestricted use, distribution, and reproduction in any medium, provided the original work is properly cited.

Paeonia lactiflora root (baishao in Chinese) is a commonly used herb in traditional Chinese medicine (TCM). Paeoniflorin (PF) and albiflorin (AF) are two major active constituents of P. lactiflora. In this paper, we aimed to investigate the hematopoietic effects of PF and $\mathrm{AF}$ on myelosuppression mice induced by radiotherapy and to explore the underlying mechanism. The finding indicated that $\mathrm{PF}$ and AF significantly increased the numbers of white blood cells (WBC) and reversed the atrophy of thymus. Furthermore, PF and AF increased the levels of granulocyte-macrophage colony-stimulating factor (GM-CSF) and interleukin-3 (IL-3) and reduced the levels of tumor necrosis factor- $\alpha$ (TNF- $\alpha$ ) in serum and increased the level of colony-stimulating factor (G-CSF) in plasma. Lastly, PF and AF not only enhanced the mRNA levels of GM-CSF and G-CSF in the spleens, but also increased the protein levels of G-CSF and GM-CSF in bone marrow. Our results suggest that PF and AF may promote the recovery of bone marrow hemopoietic function in a myelosuppressed mouse model.

\section{Introduction}

The root of Paeonia lactiflora Pall (family Ranunculaceae) is commonly used in China, Korea, and Japan. P. lactiflora is a component herb of many traditional formulae, such as SiwuTang, which has been widely used in treating palpitation, dysmenorrhea, chronic inflammation, and aminia [1]. There are a lot of major active constituents of $P$. lactiflora that have been identified, including paeoniflorin (PF), albiflorin (AF), oxypaeoniflorin, paeonilactinone, benzoyloxypaeoniflorin, and lactinolide [2]. It is well documented that PF possesses remarkable effects for pain $[3,4]$, muscle spasm $[5,6]$, inflammation $[7,8]$, and neurodegenerative disorders $[9,10]$. However, few pharmacological studies of AF were reported. Most recently, we have found that PF and AF could suppress radiation and chemotherapy-induced myelosuppression [1113]. In a recent report, an active fraction from P. lactiflora containing paeoniflorin and albiflorin (CPA) showed ameliorative effects on myelosuppression induced by radio and chemotherapy [14]. In another study, Jiang has proved the anti-inflammation effects of TGP on neutrophil cAMP-PDE activity in a rat arthritis model [6].

Blood/bone marrow system is one of the largest organs in the body that is an important potential target in ionizing radiation [15]. Acute exposure to elevated dose of ionizing radiation causes defects in hemopoiesis, resulting in low numbers of circulating blood cells, and increases susceptibility to infection [16]. Consequently, it has become a routine procedure in the investigation of hematological and bone marrow disorders in radiotherapy assessments. Nowadays, efforts to stimulate hematopoiesis in myelosuppression animals have involved hematopoietic cytokines [17], such as colony-stimulating factor (G-CSF), granulocyte-macrophage colony-stimulating factor (GM-CSF), interleukin-3 (IL-3), interleukin-6 (IL-6), and tumor necrosis factor- $\alpha$ (TNF- $\alpha$ ), that can be used to accelerate hemopoietic recovery.

Consistent with our previous study [11-13], still, we hypothesized that $\mathrm{PF}$ and $\mathrm{AF}$, two characteristic isomers 
in $P$. lactiflora, have the hematopoietic effect as well as the antimyelosuppression effect. Mechanisms associated with hematopoietic cytokines may also be involved in the hematopoietic effects of the two isomers. To test this hypothesis, we examined the effects of PF and AF in $3.5 \mathrm{~Gy} \mathrm{Co}^{60} \gamma$ rays irradiated-induced myelosuppression mice [18]. We also checked the hematopoietic functions of PF and AF, which are characterized by the changes of the number of blood cells, including WBC, RBC, and HGB. Moreover, considering the close relationship between immunity and hematopoiesis [15], the thymus index and spleen index were also investigated. To further determine the mechanism underlying the hematopoiesis activity of PF and AF, we analyzed the hematopoiesis-related cytokines in serum or in plasma as well as their mRNA in spleen and protein expressions in bone marrow cells. In this study, the effects of PF and AF on hematopoiesis in myelosuppression mice induced by radiotherapy were investigated systematically and the underlying mechanisms were also explored.

\section{Materials and Methods}

2.1. Chemical Compounds. Paeoniflorin (PF) and albiflorin (AF) were prepared in our library (patent number ZL 201110184287.4, China). The purity of PF (purity $=98.6 \%$ ) and AF (purity $=96.7 \%$ ) was measured by reverse-phase highperformance liquid chromatography (HPLC) coupled with ultraviolet detection according to Chinese Pharmacopoeia (2010, Beijing, China). The HPLC chromatogram and chemical structures of the two isomers are shown in Figure 1. PF and AF were separately dissolved in $0.9 \%$ normal saline and diluted to the desired concentration on the day of testing.

2.2. Animals. Kunming mice (male, 6 weeks old, 18-22 g) were obtained from the Vital River Co., Ltd. (Beijing, China) and maintained at controlled temperature $\left(22 \pm 2^{\circ} \mathrm{C}\right)$ and humidity $(50 \pm 10 \%)$ with a $12 \mathrm{~h}$ light/dark cycle. All efforts were made to minimize the pain of animals. The experimental protocol was approved by the Ethics Committee of Beijing University of Chinese Medicine (number Kj-dw-18-20150731$01)$.

2.3. The $\gamma$-Ray Treatment. This $\gamma$-ray treated myelosuppression mice model was set up according to Liang's method $[16,18]$. After 7 days of acclimatization, mice were divided into six groups ( $n=10$ mice per group, males), including (1) normal control group (control), (2) $\gamma$-ray treated group (model), (3) high-dose PF treatment group (PF-H, $30 \mathrm{mg} / \mathrm{kg}$ $\mathrm{PF})$, (4) L-dose PF treatment group (PF-L, $15 \mathrm{mg} / \mathrm{kg} \mathrm{PF),} \mathrm{(5)}$ high-dose AF treatment group (AF-H, $30 \mathrm{mg} / \mathrm{kg} \mathrm{AF}$ ), and (6) L-dose AF treatment group (AF-L, $15 \mathrm{mg} / \mathrm{kg} \mathrm{PF}$ ).

All mice except the control and model were intragastrically (i.g.) pretreated with the different doses of chemical compounds, PF $(30 \mathrm{mg} / \mathrm{kg}$ and $15 \mathrm{mg} / \mathrm{kg})$, and AF $(30 \mathrm{mg} / \mathrm{kg}$ and $15 \mathrm{mg} / \mathrm{kg}$ ) for 7 days. On the 8th day, mice except the normal group received total body irradiation of $3.5 \mathrm{~Gy} \mathrm{Co}^{60} \gamma$ rays at a dose rate of $1.60 \mathrm{~Gy}$. After irradiation, control group mice were treated in exactly the same way as the irradiated animals; PF and AF were administered daily intragastrically at a dose of $0.2 \mathrm{~mL} / 20 \mathrm{~g}$ body weight for 14 consecutive days. Normal and model mice were given normal saline.

2.4. Peripheral Leukocyte Count, Body Weight, Thymus Index, and Spleen Index. After the last administration, body weights of mice were measured. Then the mice were subjected to light diethyl ether anesthesia and blood was collected into clean test tubes with or without ethylenediaminetetraacetic acid (EDTA) by extracting eyeballs. A portion $(200 \mu \mathrm{L})$ of blood with EDTA $(50 \mu \mathrm{L})$ was used for peripheral hemogram analysis by Beckman Coulter Ac and T 5 full-automatic blood cell analyzer (Beckman, USA). After the mice were sacrificed, the thymus gland and spleen were excised and weighted to calculate the thymus index and spleen index: (organ weight/body weight) $\times 1000$.

2.5. Bone Morrow Histological Examination. Three femoral bones in each group were removed from the sacrificed mice and fixed in $4 \%$ paraformaldehyde. And then, bones were treated with a formic acid-sodium citrate decalcification solution for 5 days. Bones were embedded in paraffin, sectioned at $5 \mu \mathrm{m}$ for staining with $\mathrm{H} \& \mathrm{E}$, to visualize the histological examination of bone marrow.

2.6. Cytokines Levels of G-CSF, GM-CSF, IL-3, IL-6, and TNF- $\alpha$ in Plasma or Serum. Plasma or serum samples were collected from the sacrificed mice, and the levels of GCSF, GM-CSF, IL-3, IL-6, and TNF- $\alpha$ in plasma or serum were measured by Enzyme-Linked Immunosorbent Assay (ELISA) kits (Beijing Sino-UK Institute of Biological Technology, Beijing, China).

2.7. Analysis of G-CSF, GM-CSF, IL-3, $I L-6$, and TNF- $\alpha$ mRNA Expressions in Spleen. The spleen tissues were homogenized and the total RNA was extracted from the supernatant fraction. Then total RNA from each sample was reversetranscribed into cDNA using a Super RT cDNA kit (Thermo, USA), and the synthesized cDNA was used for RT-qPCR amplification using SYBR green Real-time PCR Master Mix. Furthermore, the nucleotide sequences of forward and reverse primers used for PCR are shown in Table 1. The cycling conditions were $95^{\circ} \mathrm{C}$ for $10 \mathrm{~min}$, followed by 40 cycles of $95^{\circ} \mathrm{C}$ for $15 \mathrm{~s}, 60^{\circ} \mathrm{C}$ for $60 \mathrm{~s}$, and $75^{\circ} \mathrm{C}$ for $20 \mathrm{~s}$. The RTqPCR analysis was performed with the Light Cycler 480 RTqPCR System. The results of relative expression of mRNA in each group were semiquantitated using the comparative $C_{t}$ method and calculated setting normal control as 1 .

2.8. Western Blot Analysis (G-CSF, GM-CSF, IL-3, IL-6, and TNF- $\alpha$ ) in Bone Marrow. Three mice in each group were sacrificed and then bone marrow from the femur was collected. The total protein in bone marrow was extracted with lysis buffer (50 mM Tris, pH 8.0, $150 \mathrm{mM} \mathrm{NaCl}, 0.1 \%$ sodium dodecyl sulfate, $0.5 \%$ sodium deoxycholate, $100 \mathrm{mg} / \mathrm{mL}$ phenylsulfonyl fluoride, $2 \mathrm{mg} / \mathrm{mL}$ aprotinin, $1 \mathrm{mg} / \mathrm{mL}$ pepstatin, and $10 \mathrm{mg} / \mathrm{mL}$ leupeptin), and $50 \mathrm{mg}$ protein was resolved on a $10 \%$ sodium dodecyl sulfate polyacrylamide gel. The 
TABLE 1: Primers used for quantitative RT-PCR.

\begin{tabular}{lll}
\hline Genes & Forward $\left(5^{\prime}-3^{\prime}\right)$ & Reverse $\left(5^{\prime}-3^{\prime}\right)$ \\
\hline GAPDH & AGGAGCGAGACCCCACTAACA & AGGGGGGCTAAGCAGTTGGT \\
IL-3 & GCCTGCCTACATCTGCGAAT & GGTTAGGAGAGACGGAGCCA \\
IL-6 & GTCCGGAGAGGAGACTTCAC & CTGCAAGTGCATCATCGTTGT \\
GM-CSF & TTACTTTTCCTGGGCATTGTGG & CAGGAGGTTCAGGGCTTCTTTG \\
TNF- $\alpha$ & ACCCTCACACTCACAAACCA & ATAGCAAATCGGCTGACGGT \\
G-CSF & CGCATGAAGCTAATGGGTGAGT & GACGGGTCTGAGGCACTTGTT \\
\hline
\end{tabular}

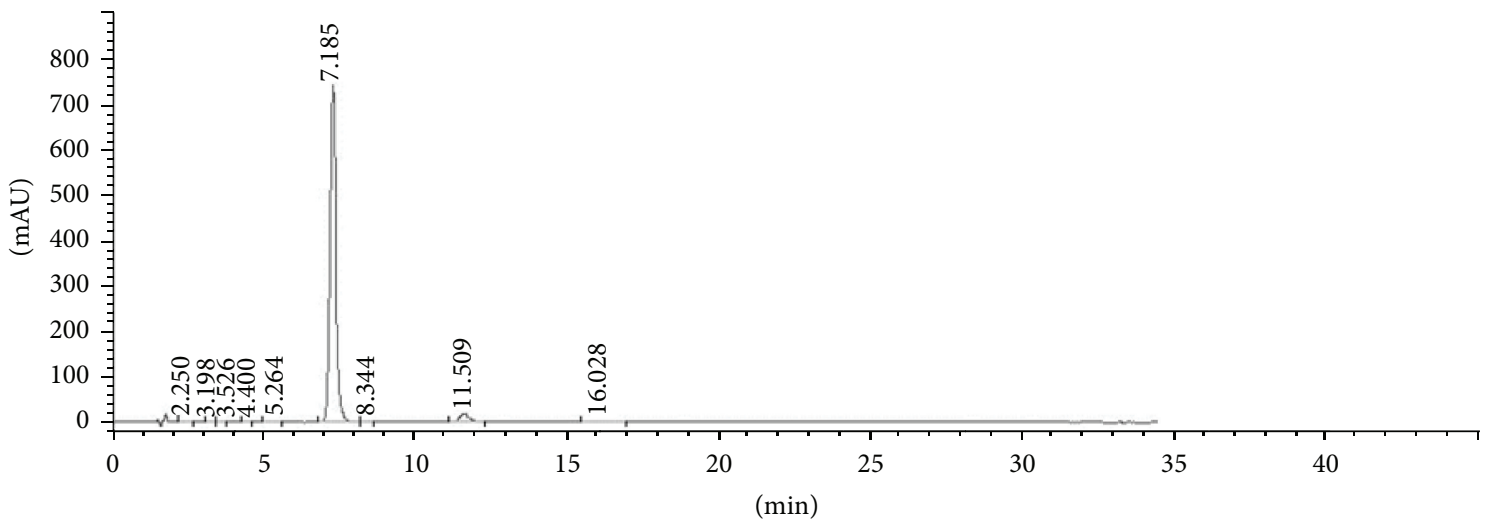

(a) Paeoniflorin (PF)

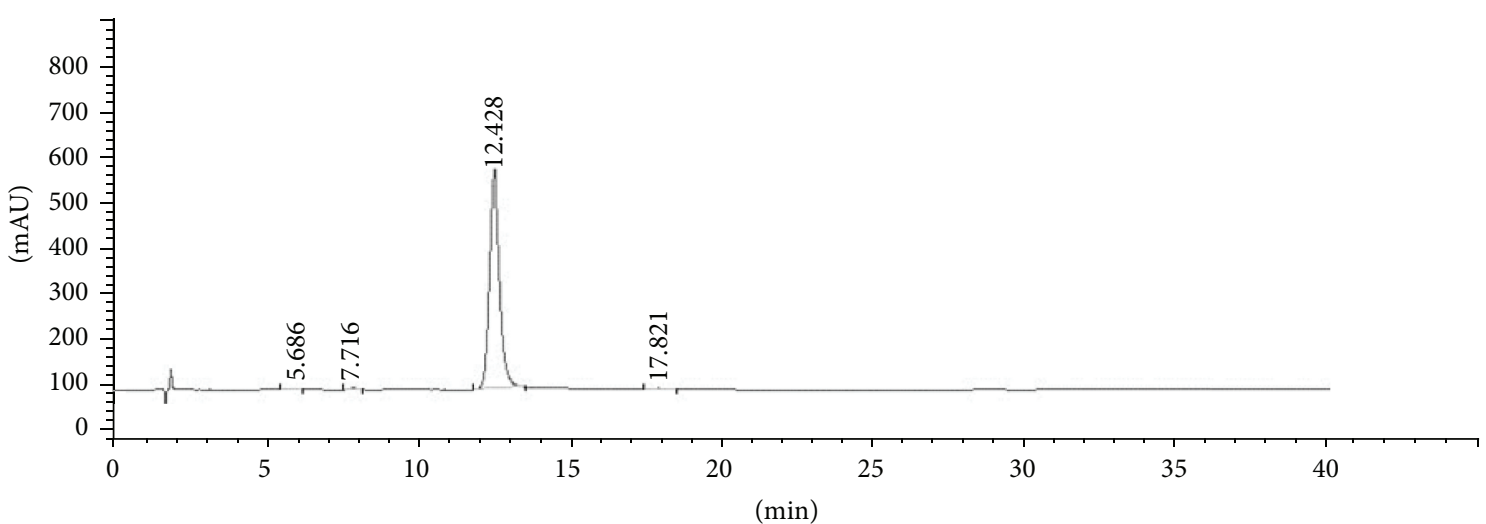

(b) Albiflorin (AF)

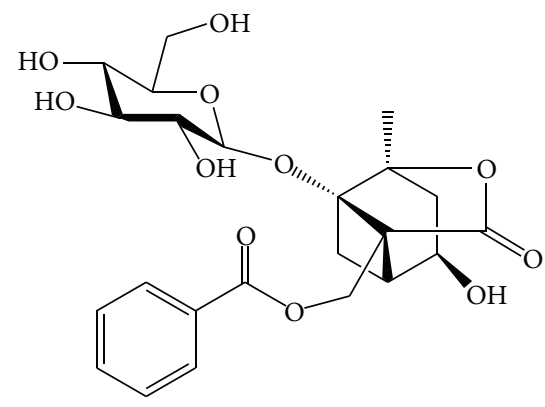

Albiflorin (AF)

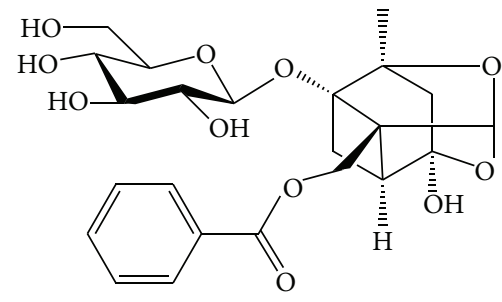

Paeoniflorin $(\mathrm{PF})$

FIGURE 1: Chemical structures and HPLC chromatograms of paeoniflorin (PF) (a) and albiflorin (AF) (b).

fractionated proteins were electrophoretically transferred to an immobilon polyvinylidene difluoride membrane and probed with antibody of G-CSF, GM-CSF, IL-3, IL-6, and TNF- $\alpha$ (Bioss. Inc., Beijing, China).
2.9. Statistical Analyses. Results are expressed as mean \pm SD. Statistical significant differences were determined by one-way analyses of variance and Student's $t$-tests. A $P$ value $<0.05$ indicates a statistically significant difference. 

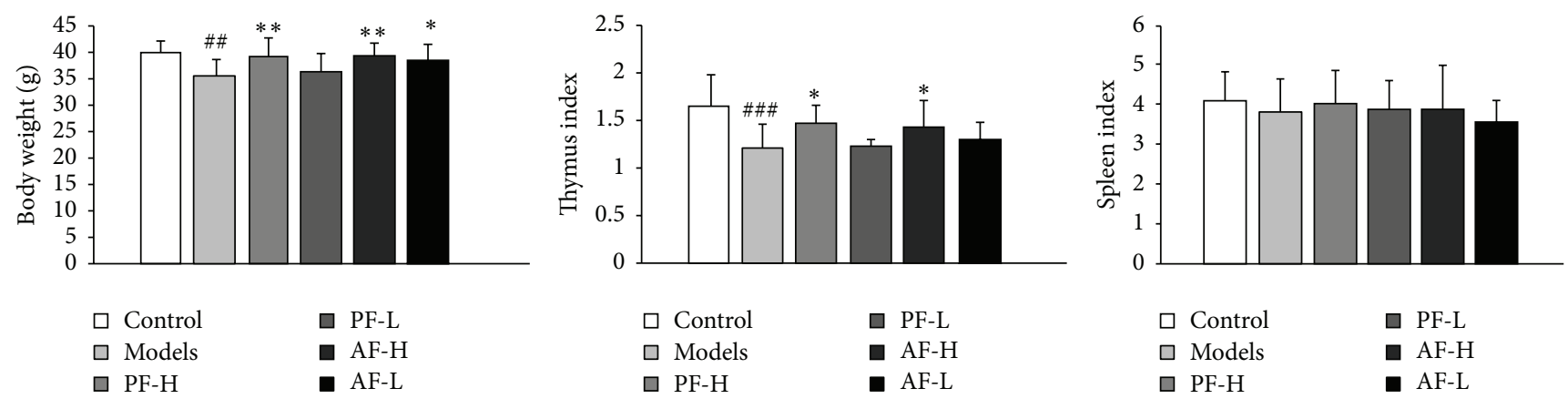

FIGURE 2: Effects of PF and AF on body weight, thymus index, and spleen index. Control = negative control (with same volume of physiologic saline). Data are expressed as means $\pm \mathrm{SD}(n=10)$. Compared with the control group: ${ }^{\# \#} P<0.01$ and ${ }^{\# \#} P<0.001$; compared with the model group: ${ }^{*} P<0.05$ and ${ }^{* *} P<0.01$.
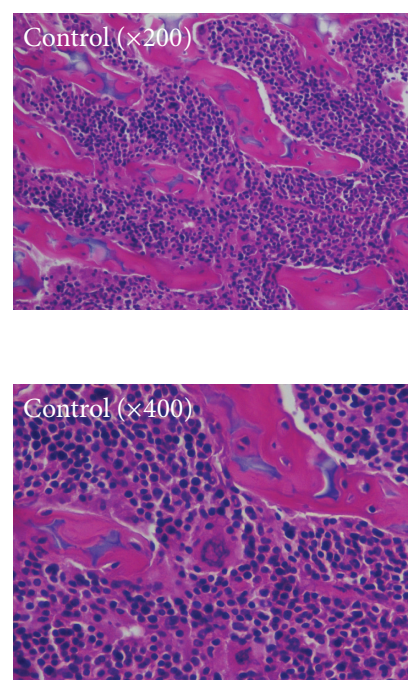
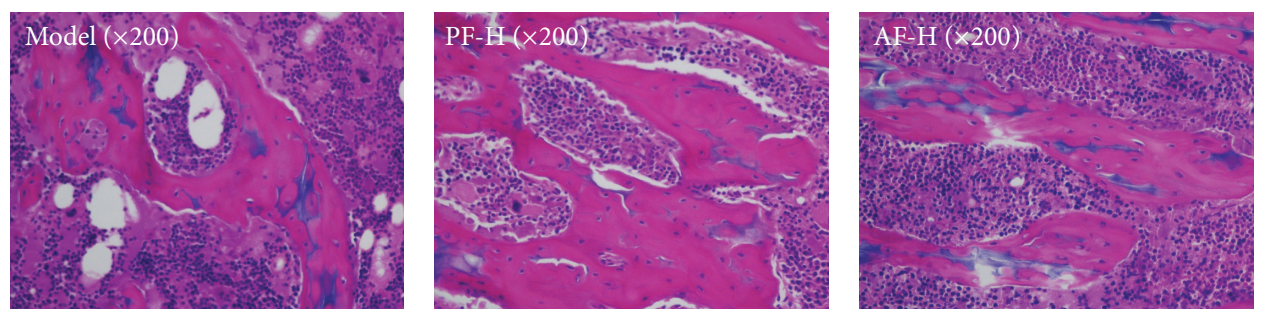

(a)
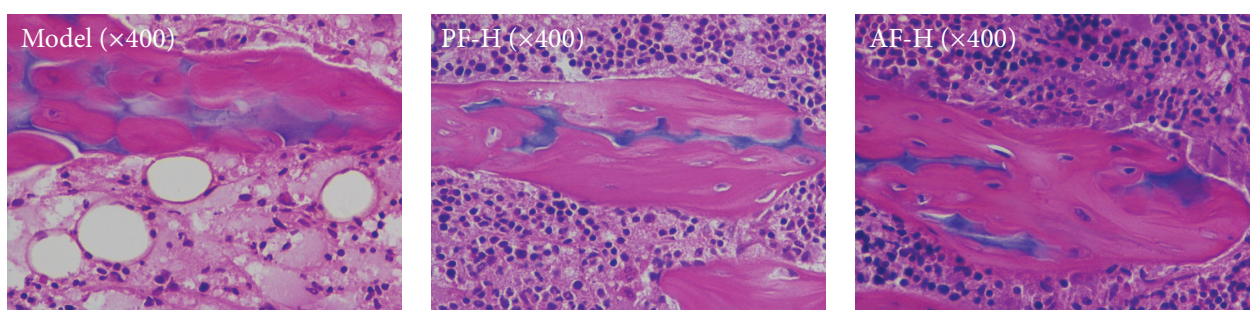

(b)

FIgURE 3: Effects of PF and AF on the bone marrow tissue histomorphology of femoral bone (H\&E staining. (a): $1 \times 200$, (b): $1 \times 400)$.

\section{Results}

3.1. Effects of PF and AF on Peripheral Blood Cells. As shown in Table 2, the number of white blood cells (WBC) in irradiation-induced model group was significantly reduced compared to that in normal group $(P<0.001)$. PF-H or AF-H treatment significantly elevated the number of WBC $(P<0.001)$. AF-L group also increased the number of WBC significantly $(P<0.01)$.

3.2. Effects of PF and AF on the Change of Body Weight, Thymus Index, and Spleen Index. As shown in Figure 2, the body weight and thymus index in model mice were significantly decreased by the irradiation treatment $(P<$ $0.01, P<0.001)$. PF-H significantly increased the body weight $(P<0.01)$ and thymus index $(P<0.05)$ and AF$\mathrm{H}$ also significantly increased the body weight $(P<0.01)$ and thymus index $(P<0.05)$. AF-L group increased the body weight $(P<0.05)$. It proved that both $\mathrm{PF}$ and $\mathrm{AF}$ could reverse the loss of body weight and the atrophy of
TABLE 2: Effects of PF and AF on peripheral blood cells (means \pm $\mathrm{SD}, n=10)$.

\begin{tabular}{lccc}
\hline Groups & WBC & RBC & HGB \\
\hline Control & $7.50 \pm 1.35$ & $2.67 \pm 0.65$ & $102.3 \pm 8.99$ \\
Model & $1.23 \pm 0.52^{\# \# \#}$ & $2.23 \pm 0.47$ & $79.2 \pm 11.33^{\# \# \#}$ \\
PF-H & $2.40 \pm 0.47^{* * *}$ & $2.36 \pm 0.96$ & $87.0 \pm 5.29$ \\
PF-L & $1.47 \pm 0.26$ & $2.24 \pm 0.53$ & $83.1 \pm 11.40$ \\
AF-H & $2.37 \pm 0.41^{* * *}$ & $2.39 \pm 0.62$ & $87.5 \pm 6.87$ \\
AF-L & $2.07 \pm 0.46^{* *}$ & $2.14 \pm 0.41$ & $84.9 \pm 10.10$ \\
\hline
\end{tabular}

Note: compared with the control group: ${ }^{\# \# \#} P<0.001$; compared with the model group: ${ }^{* *} P<0.01$ and ${ }^{* * *} P<0.001$.

hemopoietic organ (also known as immune organs) induced by irradiation. There is no change on spleen index.

3.3. Effects of PF and AF on Bone Marrow Histopathology. As shown in Figure 3, the color of the bone marrow tissue of normal mice was uniform, and the architectures of 
TABLE 3: Effects of PF and AF on the hematopoiesis-related cytokines levels (means $\pm \mathrm{SD}, n=10$ ).

\begin{tabular}{lccccc}
\hline Groups & GM-CSF & G-CSF & IL-3 & IL-6 & TNF- $\alpha$ \\
\hline Control & $115.58 \pm 16.79$ & $122.74 \pm 12.86$ & $18.26 \pm 1.43$ & $104.08 \pm 16.94$ & $56.72 \pm 7.31$ \\
Model & $74.83 \pm 13.60^{\# \# \#}$ & $85.83 \pm 15.79^{\# \# \#}$ & $11.01 \pm 1.53^{\# \# \#}$ & $154.90 \pm 24.37^{\# \# \#}$ & $70.02 \pm 11.03^{\# \#}$ \\
PF-H & $105.06 \pm 18.12^{* * *}$ & $119.31 \pm 15.98^{* * *}$ & $14.69 \pm 2.56^{* * *}$ & $130.62 \pm 33.55$ & $54.15 \pm 7.47^{* *}$ \\
PF-L & $85.41 \pm 12.36$ & $107.01 \pm 14.54^{* *}$ & $12.43 \pm 1.53$ & $131.35 \pm 32.07$ & $62.24 \pm 14.04$ \\
AF-H & $112.94 \pm 19.31^{* * *}$ & $117.12 \pm 21.86^{* * *}$ & $14.80 \pm 2.69^{* * *}$ & $129.22 \pm 34.48$ & $53.55 \pm 10.95^{* *}$ \\
AF-L & $94.27 \pm 14.61^{* *}$ & $88.90 \pm 12.72$ & $12.16 \pm 1.69$ & $152.08 \pm 26.75$ & $67.11 \pm 10.94$ \\
\hline
\end{tabular}

Note: compared with the control group: ${ }^{\# \#} P<0.01$ and ${ }^{\# \# \#} P<0.001$; compared with the model group: ${ }^{* *} P<0.01$ and ${ }^{* * *} P<0.001$.
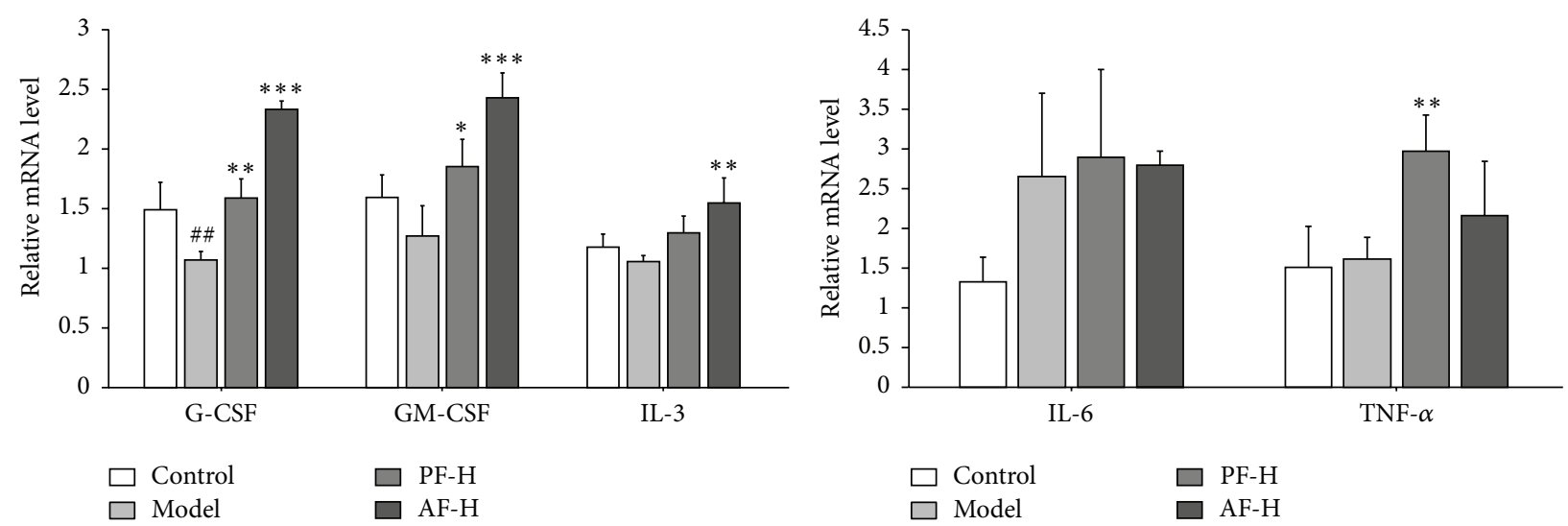

FiguRE 4: Effects of AF on GM-CSF, G-CSF, IL-3, IL-6, and TNF- $\alpha$ mRNA levels. Control = negative control (with same volume of physiologic saline). Data are expressed as means $\pm \operatorname{SD}(n=3)$. Compared with the control group: ${ }^{\# \#} P<0.01$; compared with the model group: ${ }^{*} P<0.05$, ${ }^{* *} P<0.01$, and ${ }^{* * *} P<0.001$.

periosteum, cavitas medullaris, and cartilage cells were clear, whereas, the bone marrow of model group showed a great deal of nucleated myelocytes that was reduced and replaced by vacuolation compared with that of the normal group. PF-H groups decreased the vacuole-like degradation clearly. AF-H also could increase the cell density and decrease the number of vacuole-like degradation.

3.4. Effects of PF and AF on the Hematopoiesis-Related Cytokines. As shown in Table 3, compared to the control group, serum level of GM-CSF and plasma level of G-CSF were significantly decreased after irradiation $(P<0.001$, $P<0.001)$, while levels of GM-CSF and G-CSF in PF-H $(P<0.001, P<0.001)$ and AF-H significantly increased $(P<$ $0.001, P<0.001)$, respectively. However, PF-L significantly increased G-CSF $(P<0.01)$ and AF-L increased GM-CSF $(P<0.01)$. Irradiation induced a decrease of IL-3 in serum $(P<0.001)$, and $\mathrm{PF}-\mathrm{H}$ and $\mathrm{AF}-\mathrm{H}$ administration increased the level of IL-3 compared to the model $(P<0.001, P<$ $0.001)$. As shown, irradiation also induced an increase of TNF- $\alpha$ compared to control $(P<0.01)$, and PF-H and AF$\mathrm{H}$ administration decreased the level of TNF- $\alpha$, respectively $(P<0.01, P<0.01)$.

3.5. Effects of PF and AF on G-CSF, GM-CSF, IL-3, IL-6, and TNF- $\alpha$ mRNA Levels. As shown in Figure 4, compared with the control group, the mRNA level of G-CSF was significantly decreased $(P<0.01)$ in the model animals. Compared to the model group, the mRNA level of G-CSF was elevated in PF$\mathrm{H}(P<0.01)$ and in AF-H $(P<0.001)$ treatment groups. Besides, there were no significant differences between the normal and the model mice on the mRNA levels of GM-CSF, IL-3, IL-6, and TNF- $\alpha$. PF-H increased the mRNA levels of GM-CSF and TNF- $\alpha(P<0.05, P<0.01)$. AF-H significantly increased the mRNA levels of GM-CSF and IL-3 $(P<0.001$, $P<0.01)$. The data suggested that $\mathrm{PF}-\mathrm{H}$ and AF-H could significantly enhance the mRNA levels of GM-CSF, G-CSF, and TNF- $\alpha$ in the spleens.

3.6. Effects of PF and AF on GM-CSF, G-CSF, IL-3, IL-6, and TNF- $\alpha$ Protein Levels. As shown in Figure 5, irradiation induced a decrease of G-CSF, GM-CSF, and IL-3 at protein levels compared to the control group, although there were no significant differences. PF-H could increase the levels of G-CSF, GM-CSF, and IL-3 compared to the control group, respectively $(P<0.01, P<0.001$, and $P<0.01)$ and AF$\mathrm{H}$ also could increase the levels of G-CSF, GM-CSF, and IL3 compared to the control group, respectively $(P<0.01$, $P<0.001$, and $P<0.01)$. As shown, irradiation induced increases of IL- 6 and TNF- $\alpha$ after irradiation compared to the sham irradiation $(P<0.05, P<0.01)$, PF-H could increase the levels of IL- 6 and TNF- $\alpha$ compared to model group $(P<$ $0.001, P<0.001)$, and AF-H significantly increased IL-6 and TNF- $\alpha$ levels compared to control group $(P<0.001$, $P<0.001)$. Interestingly, the IL- 6 and TNF- $\alpha$ protein levels were opposite to those in serum. 

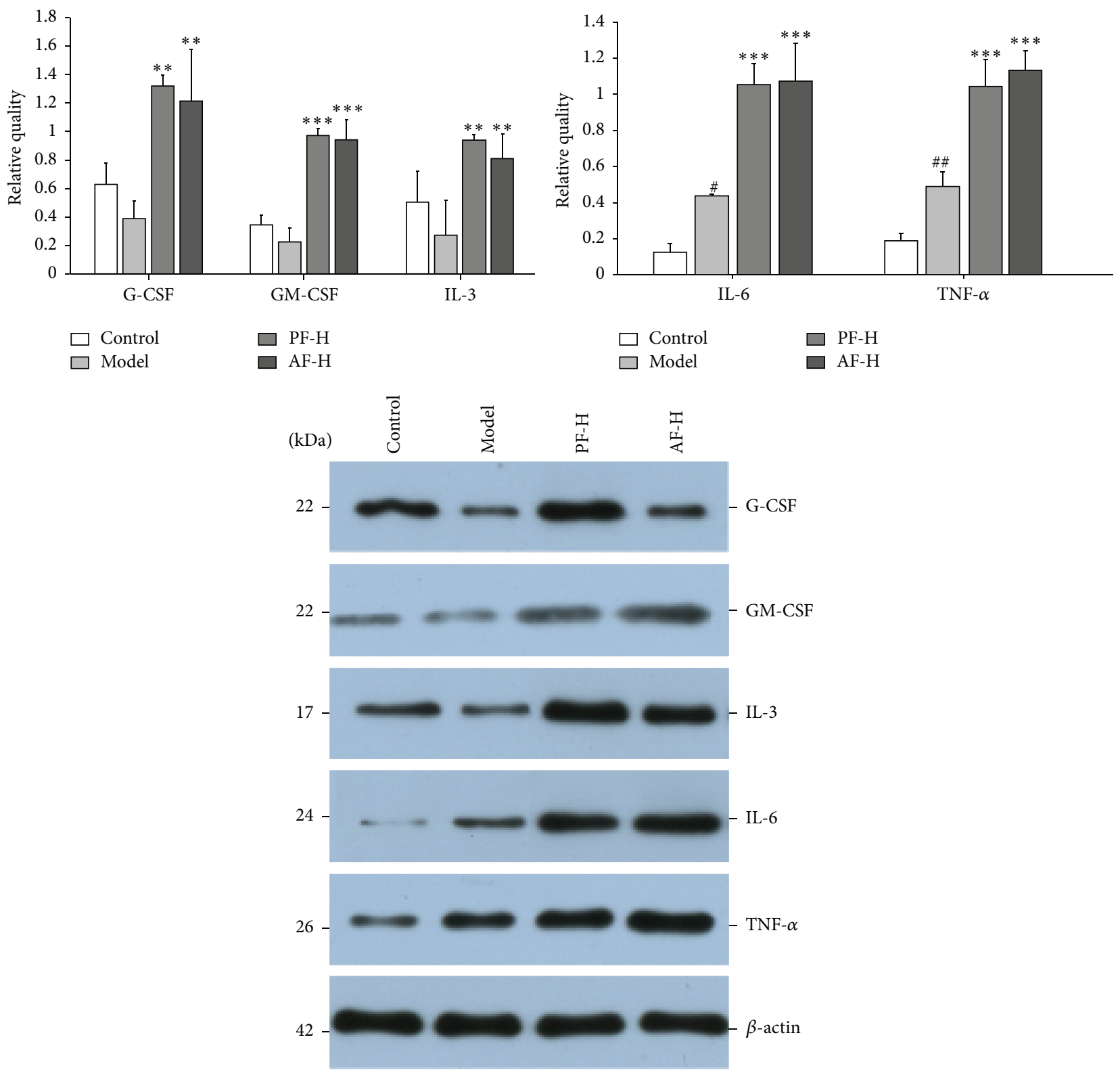

Figure 5: Effects of PF and AF on GM-CSF, G-CSF, IL-3, IL-6, and TNF- $\alpha$ protein levels. Control = negative control (with same volume of physiologic saline). Data are expressed as means $\pm \operatorname{SD}(n=3)$. Compared with the control group: ${ }^{\#} P<0.05$ and ${ }^{\# \#} P<0.01$; compared with the model group: ${ }^{* *} P<0.01$ and ${ }^{* * *} P<0.001$.

\section{Discussion}

Mice exposure to ionizing radiation leads to injury to the lymphoid and haemopoietic system, which can result in septicaemia and death [16]. Myelosuppression is a common side effect of radiotherapy and bone marrow hemopoietic tissue is the most sensitive to radiation [19]. Previous studies suggested irradiation inducing myelosuppression syndrome, capable of agent enhancing survival typically being associated with accelerated hematopoietic cytokines regeneration. Peripheral blood cell is derived from the proliferation and differentiation of hemopoietic stem cells and progenitor cells and bone marrow is in charge of the peripheral blood cells continuous replenishment [20]. In this study, the number of peripheral blood cells indirectly reflects the overall hematopoietic function. Firstly, results (Table 2) showed that $\mathrm{PF}$ and AF could significantly reverse the decrease of the quantities of WBC; the effects of PF and AF on peripheral blood cells are consistent with the previous published results of CPA [14]. Secondly, the pathological sections show that AF had improvement effects on the histopathology of bone marrow tissue (Figure 2). Thirdly, body weight was decreased in model group, while the decrease was reversed in the AFtreated mice, which means that $\mathrm{PF}$ and AF play a positive role in preventing body weight loss resulting from irradiation. Finally, it is well known that thymus and spleen are important 
hematopoietic organs besides bone marrow [21]. The results (Table 3) showed that PF and AF increased the thymus indices, which implied that $\mathrm{PF}$ and AF possess the capacity of alleviating the loss of body weight and the atrophy of hemopoietic organs (also known as immune organs) induced by irradiation besides promoting bone marrow hematopoietic function. It was exactly consistent with the previous published results of CPA or Fu fang E'jiao Jiang (a famous TCM formula, which has widely been used in treating anemia) $[14,16]$. The results demonstrate that PF and AF also have protection effects on the immune conditions against the side effects caused by radiotherapy.

In addition, hematopoiesis is a diverse process, regulated by various hematopoietic cytokines, such as G-CSF, GM-CSF, IL-3, IL- 6 , and TNF- $\alpha$. A key feature of enhancing recovery from the radiation syndrome is the hematopoietic cytokines activation [22]. In this study, both PF and AF improved the G-CSF level in plasma, mRNA level in spleen, and protein level in bone marrow cells. The effect on G-CSF was consistent with the previous published results demonstrating the effect of Siwu-Tang in anaemia model by irradiation [16]. We also analyzed the contents of PF and AF in SiwuTang by high-performance liquid chromatography (HPLC) analysis, which showed the presence of $\mathrm{PF}$ and $\mathrm{AF}$, which showed the presence of PF and AF to be $6.4 \%$ and 3.3\%, respectively. Although the activities of a single compound might not show the same activities of a formula, our results suggest that the majority of effective ingredients in SiwuTang are PA and AF. Moreover, both PF and AF increased the levels of GM-CSF and IL-3 in serum and increased the protein levels in bone barrow cells, and AF enhanced the IL3 mRNA levels in spleen. The reason may be that PF and AF regulated the GM-CSF and IL-3 to stimulate proliferation and maturation of granulocyte and macrophage myeloid cells [23] and accelerate the recovery of circulating hematopoietic lineage and stimulate the growth and effector functions of lymphocytes and macrophages [24]. Furthermore, TNF- $\alpha$ is a potent inhibitor, which in turn affects the differentiation of early bone marrow progenitor cells by altering their response to CSFs [25] and accelerating the release of granulocytes from bone marrow [26]. Interestingly, our present study revealed that both PF and AF could enhance the mRNA level and protein level, while the protein levels of TNF- $\alpha$ were opposite to those in serum, and the reason needs to be investigated further. However, IL- 6 is produced by a number of normal and transformed cell lines [27], which can either promote or inhibit the growth of tumor cells. Importantly, IL-6 acts in concert with IL-3 to induce multilineage progenitors from murine spleens [28]. However in our study, no significant activation of IL-6 was observed. Similar results showed that Rg1 (a chemical compound in Panax ginseng, which is traditionally used as a restorative, antidiabetic, antivomiting, and anticancer agent worldwide) or Fu fang E'jiao Jiang (Panax ginseng is a component herb of this formula) changed the expression of hematopoiesis-related cytokines in animal models [19, 27]. PF and AF promoted hematopoiesis probably through stimulating the expression of GM-CSF, IL-3, and G-CSF in myelosuppression mice induced by irradiation. Further experiments are necessary to clarify their mechanism of action. The effect of PF and AF on other hematopoiesisrelated cytokines remains to be explored further in the ongoing study.

\section{Conclusions}

In conclusion, the results of the present study indicate that $\mathrm{PF}$ and AF have potent ameliorative effects on radiationinduced myelosuppression mice. $\mathrm{PF}$ and $\mathrm{AF}$ stimulate the hematopoiesis-related cytokines and enhanced hematopoietic progenitor cell activity resulting in accelerated blood cell recovery. Additionally, further pharmacological studies to determine more mechanisms underlying the therapeutic effects of PF and AF are needed.

\section{Competing Interests}

The authors have no conflict of interests to declare.

\section{Acknowledgments}

The authors are grateful for financial support from National Nature Science Foundation of China (Project nos. 81473370 and 81173569).

\section{References}

[1] L. L. L. Yeh, J.-Y. Liu, Y.-S. Liu, K.-S. Lin, T.-F. Tsai, and L.H. Wang, "Anemia-related hemogram, uterine artery pulsatility index, and blood pressure for the effects of four-agentsdecoction (Si Wu Tang) in the treatment of primary dysmenorrhea," Journal of Alternative and Complementary Medicine, vol. 15 , no. 5, pp. 531-538, 2009.

[2] National Committee of Pharmacopoeia, Pharmacopoeia of the People's Republic of China (Part one), Chemical Industry Press, Beijing, China, 2015.

[3] H.-Y. Yu, D.-G. Mu, J. Chen, and W. Yin, "Suppressive effects of intrathecal paeoniflorin on bee venom-induced pain-related behaviors and spinal neuronal activation," Pharmacology, vol. 88, no. 3-4, pp. 159-166, 2011.

[4] C. Jiang, L. Xu, L. Chen et al., "Selective suppression of microglial activation by paeoniflorin attenuates morphine tolerance," European Journal of Pain, vol. 19, no. 7, pp. 908-919, 2015.

[5] K.-N. Nam, C. G. Yae, J.-W. Hong, D.-H. Cho, J. H. Lee, and E. H. Lee, "Paeoniflorin, a monoterpene glycoside, attenuates lipopolysaccharide-induced neuronal injury and brain microglial inflammatory response," Biotechnology Letters, vol. 35, no. 8, pp. 1183-1189, 2013.

[6] D. Jiang, Y. Chen, X. Hou, J. Xu, X. Mu, and W. Chen, "Influence of Paeonia lactiflora roots extract on cAMP-phosphodiesterase activity and related anti-inflammatory action," Journal of Ethnopharmacology, vol. 137, no. 1, pp. 914-920, 2011.

[7] M. Zhong, W.-L. Song, Y.-C. Xu, Y. Ye, and L.-Y. Feng, "Paeoniflorin ameliorates ischemic neuronal damage in vitro via adenosine $A_{1}$ receptor-mediated transactivation of epidermal growth factor receptor," Acta Pharmacologica Sinica, vol. 36, no. 3, pp. 298-310, 2015.

[8] R.-B. Guo, G.-F. Wang, A.-P. Zhao, J. Gu, X.-L. Sun, and G. Hu, "Paeoniflorin protects against ischemia-induced brain damages 
in rats via inhibiting MAPKs/NF- $\kappa \mathrm{B}$-mediated inflammatory responses," PLoS ONE, vol. 7, no. 11, article e49701, 2012.

[9] F.-M. Qiu, X.-M. Zhong, Q.-Q. Mao, and Z. Huang, "Antidepressant-like effects of paeoniflorin on the behavioural, biochemical, and neurochemical patterns of rats exposed to chronic unpredictable stress," Neuroscience Letters, vol. 541, pp. 209-213, 2013.

[10] F.-M. Qiu, X.-M. Zhong, Q.-Q. Mao, and Z. Huang, "The antidepressant-like effects of paeoniflorin in mouse models," Experimental and Therapeutic Medicine, vol. 5, no. 4, pp. 11131116, 2013.

[11] S.-S. Qu, J.-J. Zhang, Y.-F. Huang, W. Li, Y.-L. Zhu, and Q. Yang, "Study on blood enriching effects of $\gamma$-ray radiation of paeoniflorin and albiflorin on mouse model of blood deficiency," Zhongguo Zhong Yao Za Zhi, vol. 39, no. 15, pp. 29522955, 2014.

[12] Y.-L. Zhu, J.-J. Zhang, J.-X. Wang et al., "Comparative study on effects of blood enriching on mouse model of blood deficiency syndrome induced by cyclophosphamide of albiflorin, paeoniflorin on levels of GM-CSF, IL-3 and TNF- $\alpha$," Zhongguo Zhong Yao Za Zhi, vol. 40, no. 2, pp. 330-333, 2015.

[13] Y.-L. Zhu, J.-J. Zhang, Y.-F. Huang et al., "Comparative study on effects of blood enriching on mouse model of blood deficiency syndrome induced by cyclophosphamide of White Peony Root, Red Peony Root on levels of IL-3 and TNF- $\alpha$," China Journal of Traditional Chinese Medicine and Pharmacy, vol. 4, no. 29, pp. 1058-1060, 2014.

[14] W. Xu, L. Zhou, X. Ma et al., “Therapeutic effects of combination of paeoniflorin and albiflorin from Paeonia radix on radiation and chemotherapy-induced myelosuppression in mice and rabbits," Asian Pacific Journal of Cancer Prevention, vol. 12, no. 8, pp. 2031-2037, 2011.

[15] M. Kawakatsu, Y. Urata, R. Imai et al., "Nicaraven attenuates radiation-induced injury in hematopoietic stem/progenitor cells in mice," PLoS ONE, vol. 8, no. 3, article e60023, pp. 1-7, 2013.

[16] Z.-C. Ma, Q. Hong, Y.-G. Wang et al., "Effects of ferulic acid on hematopoietic cell recovery in whole-body gamma irradiated mice," International Journal of Radiation Biology, vol. 87, no. 5, pp. 499-505, 2011.

[17] R. Arora, D. Gupta, R. Chawla et al., "Radioprotection by plant products: present status and future prospects," Phytotherapy Research, vol. 19, no. 1, pp. 1-22, 2005.

[18] Q.-D. Liang, Y. Gao, H.-L. Tan et al., "Effects of four Si-WuTang's constituents and their combination on irradiated mice," Biological and Pharmaceutical Bulletin, vol. 29, no. 7, pp. 13781382, 2006.

[19] M. Liu, H. Tan, X. Zhang et al., "Hematopoietic effects and mechanisms of Fufang E'jiao Jiang on radiotherapy and chemotherapy-induced myelosuppressed mice," Journal of Ethnopharmacology, vol. 152, no. 3, pp. 575-584, 2014.

[20] P. J. Carey, "Drug-induced myelosuppression: diagnosis and management," Drug Safety, vol. 26, no. 10, pp. 691-706, 2003.

[21] M. Lei, J. Wang, Y. Wang et al., "Study of the radio-protective effect of cuttlefish ink on hemopoietic injury," Asia Pacific Journal of Clinical Nutrition, vol. 16, no. 1, pp. 239-243, 2007.

[22] V. K. Singh, M. B. Grace, V. I. Parekh, M. H. Whitnall, and M. R. Landauer, "Effects of genistein administration on cytokine induction in whole-body gamma irradiated mice," International Immunopharmacology, vol. 9, no. 12, pp. 1401-1410, 2009.

[23] B. Saha, A. Saini, R. Germond, P. J. Perrin, D. M. Harlan, and T. A. Davis, "Susceptibility or resistance to Leishmania infection is dictated by the macrophages evolved under the influence of IL-3 or GM-CSF," European Journal of Immunology, vol. 29, no. 7, pp. 2319-2329, 1999.

[24] M. Zhang, L. Yin, K. Zhang et al., "Response patterns of cytokines/chemokines in two murine strains after irradiation," Cytokine, vol. 58, no. 2, pp. 169-177, 2012.

[25] L. Lu, M. C. Heinrich, L.-S. Wang et al., "Retroviral-mediated gene transduction of c-kit into single hematopoietic progenitor cells from cord blood enhances erythroid colony formation and decreases sensitivity to inhibition by tumor necrosis factor- $\alpha \alpha$ and transforming growth factor- $\beta 1$," Blood, vol. 94, no. 7, pp. 2319-2332, 1999.

[26] C. Caux, B. Vanbervliet, C. Massacrier, I. Durand, and J. Banchereau, "Interleukin-3 cooperates with tumor necrosis factor $\alpha$ for the development of human dendritic/Langerhans cells from cord blood CD34+ hematopoietic progenitor cells," Blood, vol. 87, no. 6, pp. 2376-2385, 1996.

[27] P. M. Bartold and D. R. Haynes, "Interleukin- 6 production by human gingival fibroblasts," Journal of Periodontal Research, vol. 26, no. 4, pp. 339-345, 1991.

[28] H. R. B. Raghavendran, R. Sathyanath, J. Shin et al., "Panax ginseng modulates cytokines in bone marrow toxicity and myelopoiesis: ginsenoside RG1 partially supports myelopoiesis," PLoS ONE, vol. 7, no. 4, Article ID e33733, pp. 1-14, 2012. 


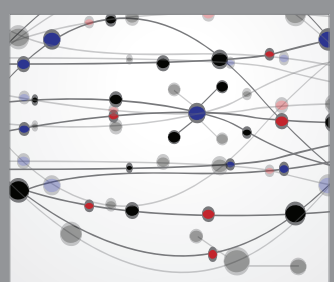

The Scientific World Journal
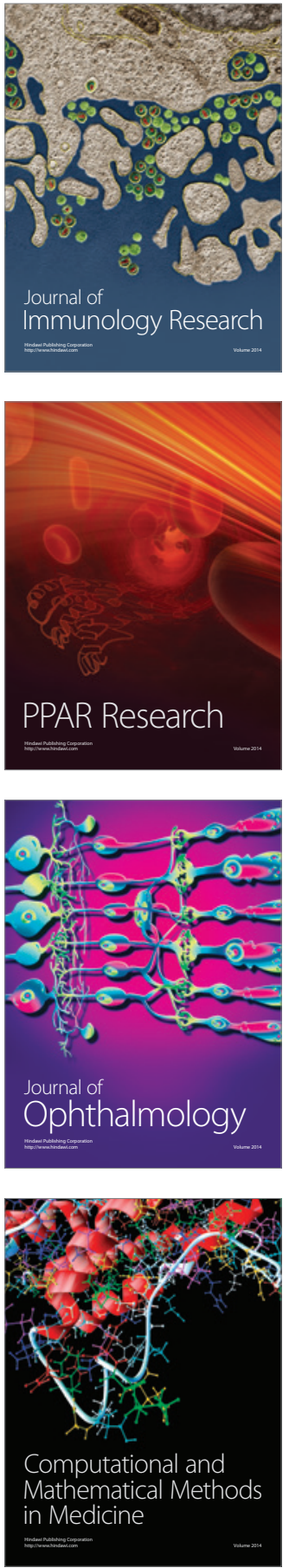

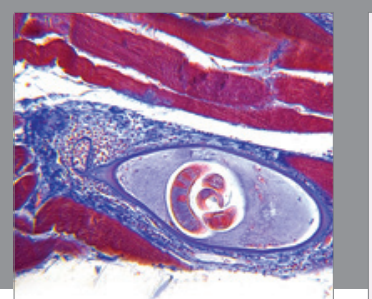

Gastroenterology Research and Practice

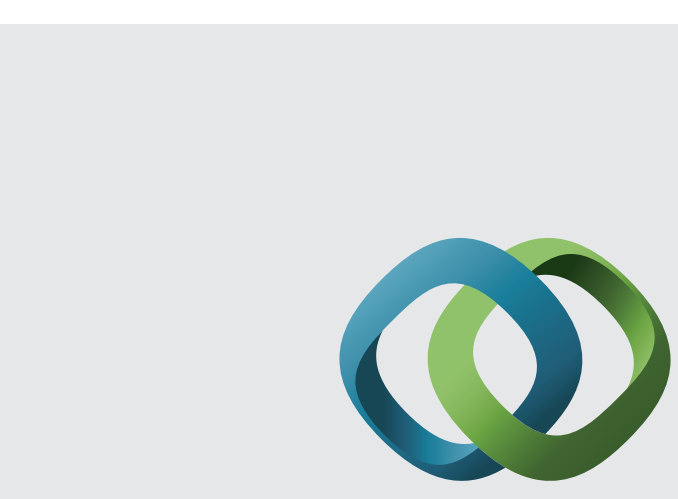

\section{Hindawi}

Submit your manuscripts at

http://www.hindawi.com
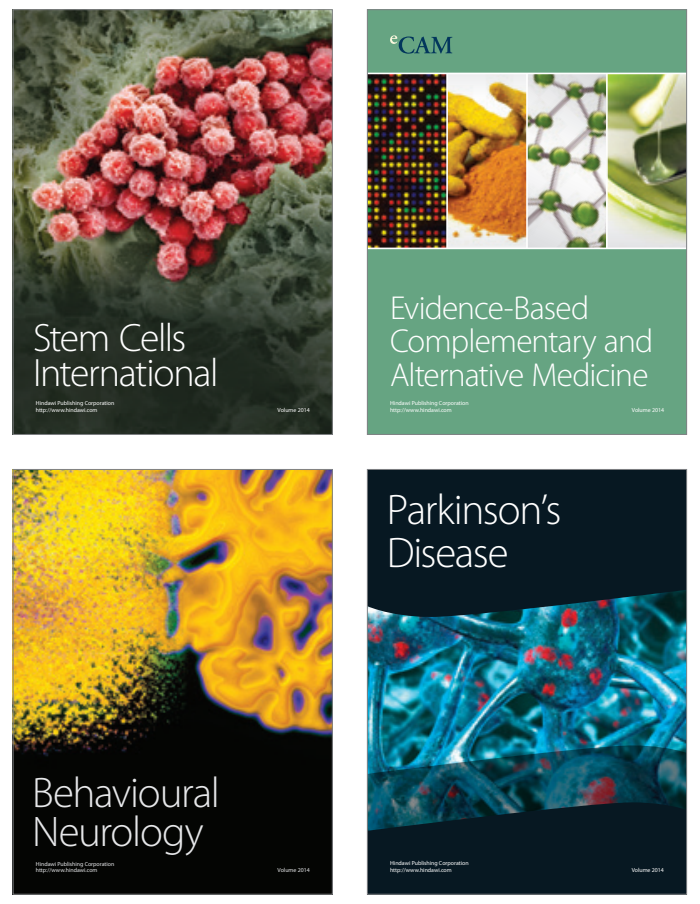
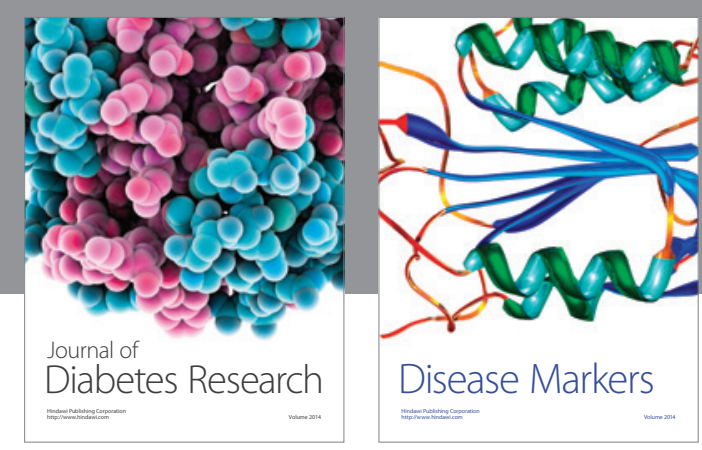

Disease Markers
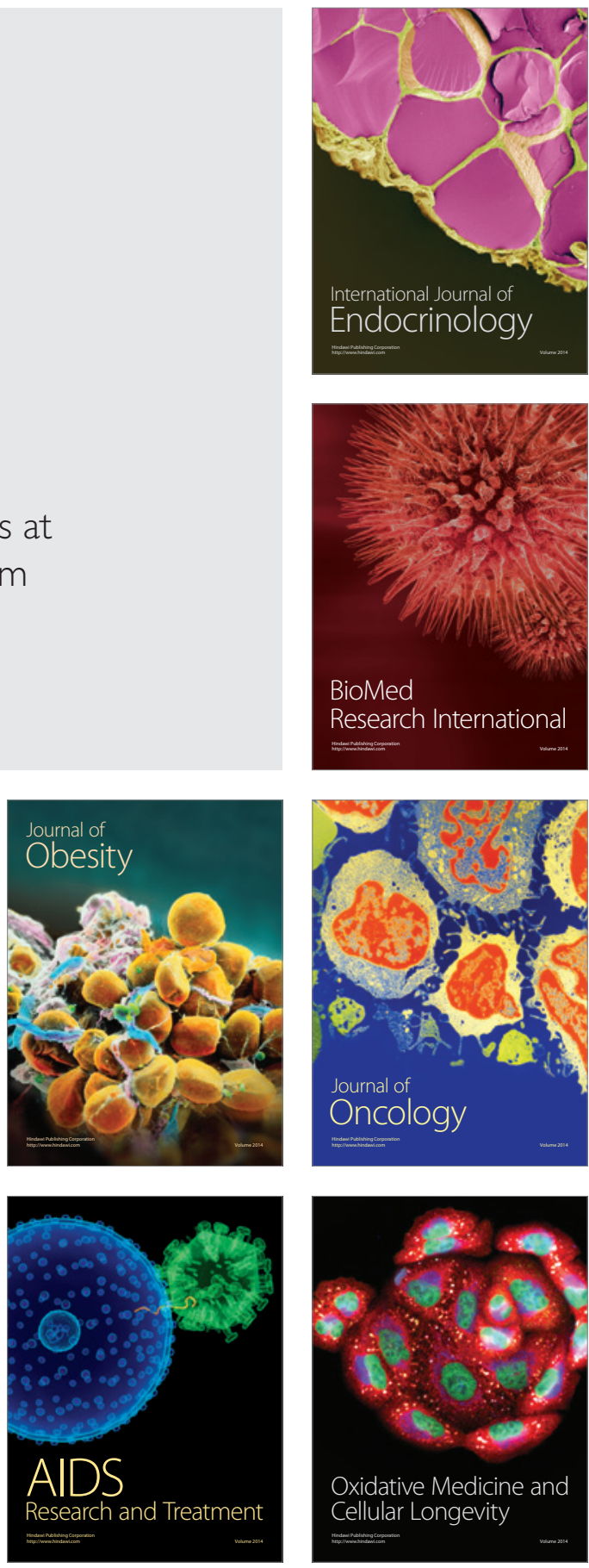